\title{
ANÁLISE DE SENSIBILIDADE GLOBAL E SELEÇÃO DE PARÂMETROS DO MODELO CINÉTICO DE HIDRÓLISE ENZIMÁTICA DA PALHA DE CANA-DE-AÇÚCAR
}

\author{
O. IVO ${ }^{1}$, K. P. F. ALBERTON ${ }^{1}$, J. D. A, MARTÍNEZ ${ }^{1}$, A. R. SECCHI ${ }^{1}$ \\ ${ }^{1}$ Universidade Federal do Rio de Janeiro, Programa de Engenharia Química \\ E-mail para contato: arge@peq.coppe.ufrj.br
}

\begin{abstract}
RESUMO - Para tratar do problema de estimação de parâmetros com escassez de dados experimentais, foi feita a análise de sensibilidade global de um modelo cinético que descreve a hidrólise enzimática da palha de cana-de-açúcar prétratada hidrotermicamente e, a partir dos resultados obtidos, foi realizada uma ordenação da influência dos parâmetros no modelo atentando-se às variâncias condicionadas e à existência de correlações paramétricas.
\end{abstract}

\section{INTRODUÇÃO}

Resíduo agroindustrial gerado a partir do desfolhamento da cana-de-açúcar durante a colheita, a palha está entre as matérias-primas renováveis com maior potencial para produção de etanol lignocelulósico. Estima-se que o reaproveitamento de 50\% deste resíduo, considerando rendimentos de apenas 50\% nas etapas de beneficiamento, leve a um aumento de $20 \%$ na produção de etanol (Martínez, 2014). Além disso, a produção de etanol utilizando a palha da cana-de-açúcar mitiga os graves impactos ambientais resultantes da queima deste resíduo, atualmente proibida pelos órgãos legislativos ambientais.

Dentre os processos de produção de etanol a partir de biomassa, o processo enzimático tem sido considerado a tecnologia mais promissora: (i) as enzimas são altamente específicas (Béguin e Aubert, 1994), (ii) os produtos da reação usualmente são açúcares redutores, (iii) apresenta baixo custo com utilidades, pois a enzima atua em condições brandas, i.e., pH 4,8 e temperatura entre $45-50{ }^{\circ} \mathrm{C}$ (Sun e Cheng, 2002), (iv) permite maiores rendimentos, e (v) possui baixo custo de manutenção. Entretanto, alguns desafios devem ser superados para sua reprodução em escala industrial: (i) a lenta velocidade de reação, (ii) o alto custo das enzimas (Hahn-Hägerdal et al., 2006), e (iii) a dificuldade em realizar a hidrólise enzimática em concentrações de substrato, superiores a 12-15\% m/m (Hodge et al., 2009). A hidrólise enzimática de biomassa lignocelulósica ainda é uma etapa crítica para a viabilidade do processo de conversão pela via bioquímica e a geração e análise de dados experimentais é uma tarefa trabalhosa, demorada e analiticamente desafiadora (Kadam et al., 2004). Desta forma, tem-se um grande apelo ao uso da modelagem matemática, a fim de obter maior compreensão dos fenômenos envolvidos, bem como ferramenta de apoio ao projeto, otimização e controle deste processo complexo.

Neste contexto, este trabalho apresenta a análise de sensibilidade global do modelo cinético de hidrólise da palha de cana-de-açúcar desenvolvido por Kadam et al. (2004). Para a análise de sensibilidade global, empregam-se os índices de sensibilidade global - ISG (Sobol, 
1991), que apresenta o menor custo computacional nesta classe de ferramentas. Como resultados, além de verificar a sensibilidade do modelo ao comportamento do processo, foi possível identificar o conjunto de parâmetros mais significativos para futura estimação, bem como obter a melhor região experimental para sua estimação.

\section{MODELO MATEMÁTICO}

As Equações 1 a 15 apresentam o modelo matemático que descreve a cinética da hidrólise enzimática da palha da cana-de-açúcar (Kadam et al., 2004). A descrição dos elementos deste modelo é apresentada pelo Apêndice, item 6 deste artigo.

Isoterma de adsorção de Lagmuir

$E_{B S}=\frac{E_{B}}{S}=\frac{E_{\max } K_{a d} E_{F}}{1+K_{a d} E_{F}}$

Enzima adsorvida em celulose:

$E_{B C}=E_{B} \frac{C}{S}$

Enzima adsorvida em hemicelulose

$$
E_{B H}=E_{B} \frac{H}{S}
$$

Quantidade total de enzimas

$$
E_{T}=E_{F}+E_{B}
$$

Quantidade total de sólidos

$S=C+H+L$

Reatividade dos substratos

$R_{S}=\alpha \frac{S}{S_{0}}$

Taxa de conversão de celulose para celobiose $\quad r_{1}=\frac{k_{1 r} E_{B C} R_{S} S}{1+\frac{G 2}{K_{1 I G 2}}+\frac{G}{K_{1 I G}}+\frac{X}{K_{1 I X}}}$

Taxa de conversão de celulose em glicose

$$
r_{2}=\frac{k_{2 r} E_{B C} R_{S} S}{1+\frac{G 2}{K_{2 I G 2}}+\frac{G}{K_{2 I G}}+\frac{X}{K_{2 I X}}}
$$

Taxa de conversão de celobiose em glicose

$$
r_{3}=\frac{k_{3 r} E_{F} G 2}{K_{3 M}\left(1+\frac{G}{K_{3 I G}}+\frac{X}{K_{3 I X}}\right)+G 2}
$$

Taxa de conversão de hemicelulose em xilose

$$
r_{4}=\frac{k_{4 r} E_{B H} R_{S} S}{1+\frac{G 2}{K_{4 I G 2}}+\frac{G}{K_{4 I G}}+\frac{X}{K_{4 I X}}}
$$

Balanço de massa de celulose

$$
\frac{d C}{d t}=-r_{1}-r_{2}, \quad C(0)=24,45 g / L
$$

Balanço de massa de celobiose

$$
\frac{d G 2}{d t}=1,056 r_{1}-r_{3}, \quad G 2(0)=0,0 g / L
$$

Balanço de massa de glicose

$$
\frac{d G}{d t}=1,111 r_{2}+1,053 r_{3}, \quad G(0)=0,0 g / L
$$


Balanço de massa de hemicelulose

$$
\frac{d H}{d t}=-r_{4}, \quad H(0)=11,25 g / L
$$

Balanço de massa de xilose

$$
\frac{d X}{d t}=1,136 r_{4}, \quad X(0)=0,0 g / L
$$

\section{ANÁLISE DE SENSIBILIDADE GLOBAL}

Em se tratando de análise de sensibilidade, uma dificuldade natural da abordagem local é a necessidade de uma boa estimativa inicial dos fatores do modelo a serem avaliados (variáveis e parâmetros). Na prática, é muito comum a falta de informação confiável sobre os fatores do modelo, sobretudo em relação aos parâmetros. Desta forma, a análise de sensibilidade global se mostra uma opção bastante atraente com propriedades tais como: independência do modelo, captura da influência da faixa de variação dos fatores, avaliação dos efeitos de interação entre os fatores e possibilidade de tratamento dos fatores individualmente (Saltelli et al., 2008). Na abordagem global, destaca-se os chamados índices de sensibilidade, IS, cujo cálculo consiste em um procedimento numérico baseado em técnicas de amostragem Monte Carlo (Sobol, 1991). O IS de primeira ordem $\left(S_{i}\right)$ indica o quanto, em média, se pode reduzir a variância da saída se este fator $\left(\right.$ Par $\left._{i}\right)$ for fixado; $\operatorname{logo}, S_{i}$ é a medida do efeito principal (Equação 16). De acordo com Saltelli et al. (2008), estas estimativas $\left\{S_{i}, i=1,2, \ldots, k\right\}$ apresentam um custo computacional de $C=N(k+2)$, sendo $N$ o número de gerações e $k$ o número de parâmetros.

$$
S_{i}=\frac{V\left[E\left(Y / \text { Par }_{i}\right)\right]}{V(Y)}
$$

em que $V\left[E\left(Y /\right.\right.$ Par $\left.\left._{i}\right)\right]$ é a variância condicional de $Y$ para um dado $\operatorname{Par}_{i}$ e $V(Y)$ representa a variância incondicional (total) de $Y$.

\section{RESULTADOS}

Estabelecidos previamente na literatura (Kadam et al., 2004; Angarita, 2014), os parâmetros $K_{a d}, E_{\max }$ e $\alpha$ não foram analisados, tendo seus valores fixados respectivamente em 8,577 g de proteína/g de solução, $0,026 \mathrm{~g}$ de enzima/ $\mathrm{kg}$ de substrato e 1 (admensional). Para os demais parâmetros do modelo, o $S_{i}$ foi calculado com um número de gerações igual $1 \times 10^{5}$, assumindo distribuição normal de probabilidades, média $(\boldsymbol{\mu})$ igual a estimativa inicial (Câmara, 2012) e variância ( $\boldsymbol{\sigma}$ ) igual a $25 \%$ deste valor; como apresentado pela Tabela 1. O modelo matemático e demais procedimentos numéricos foram implementados em MatLab ${ }^{\circledR}$ 2014, sendo as gerações obtidas por meio do comando randnorm.

Foram calculados os $S_{i}$ de cada parâmetro, ao longo dos perfis temporais das variáveis de resposta do modelo. Deste modo, o ordenamento dos parâmetros quanto a influência sobre a predição para o cenário experimental estudado é dado por: $k_{2 r}, K_{2 I G}, k_{4 r}, K_{4 I G}, k_{3 r}, k_{1 r}, K_{1 I G}$, $K_{3 M}$, sendo os parâmetros $k_{3 r}$ e $k_{l r}$ de igual influência. A Figura 1 apresenta o comportamento 
dos $S_{i}$ desses 8 parâmetros dentre os 16 do modelo matemático, pois possuem maior influência sobre a predição do modelo, considerando o cenário experimental estudado.

Tabela 1 - Média $(\boldsymbol{\mu})$ e desvio padrão $(\boldsymbol{\sigma})$ dos parâmetros.

\begin{tabular}{ccc|ccc}
\hline Parâmetro & $\boldsymbol{\mu}$ & $\boldsymbol{\sigma}$ & Parâmetro & $\boldsymbol{\mu}$ & $\boldsymbol{\sigma}$ \\
\hline$k_{l r}$ & 0,650 & 0,130 & $k_{3 r}$ & 254,5 & 50,9 \\
$K_{I I G 2}$ & 9,830 & 1,966 & $K_{3 M}$ & 24,5 & 4,9 \\
$K_{I I G}$ & 0,176 & 0,035 & $K_{3 I G}$ & 41,75 & 8,35 \\
$K_{1 I X}$ & 9,540 & 1,908 & $K_{3 I X}$ & $1,0 \times 10^{10}$ & $2,0 \times 10^{9}$ \\
$k_{2 r}$ & 3,170 & 0,634 & $k_{4 r}$ & 57,79 & 11,558 \\
$K_{2 I G 2}$ & $1,0 \times 10^{10}$ & $2,0 \times 10^{9}$ & $K_{4 I G 2}$ & $1,0 \times 10^{10}$ & $2,0 \times 10^{9}$ \\
$K_{2 I G}$ & 0,15 & 0,03 & $K_{4 I G}$ & 0,0230 & 0,0046 \\
$K_{2 I X}$ & 8,790 & 1,758 & $K_{4 I X}$ & 9,810 & 1,962 \\
\hline
\end{tabular}

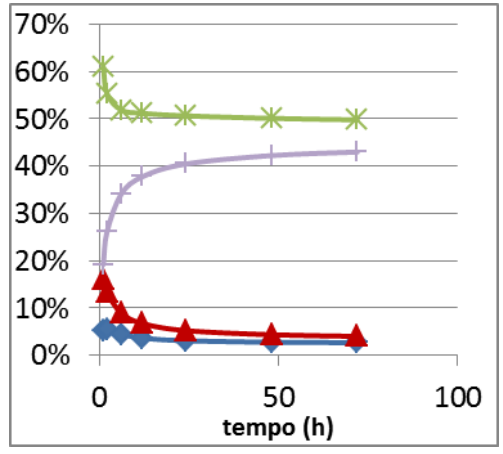

(a)

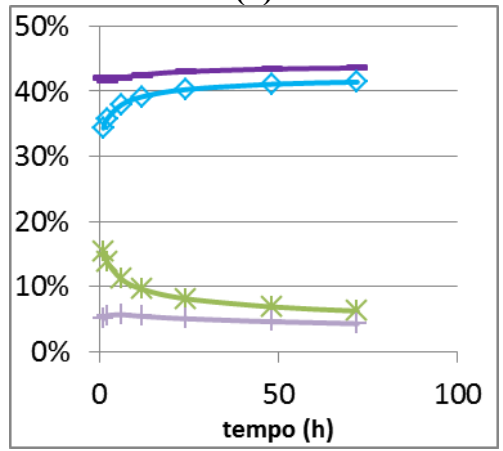

(d)

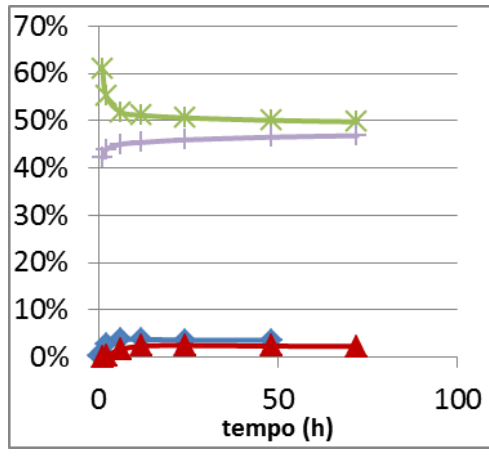

(b)

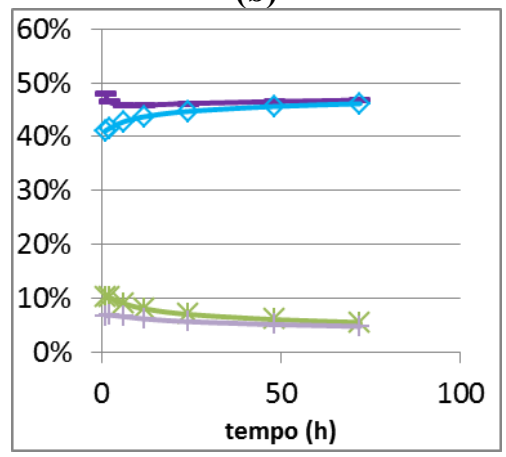

(e)

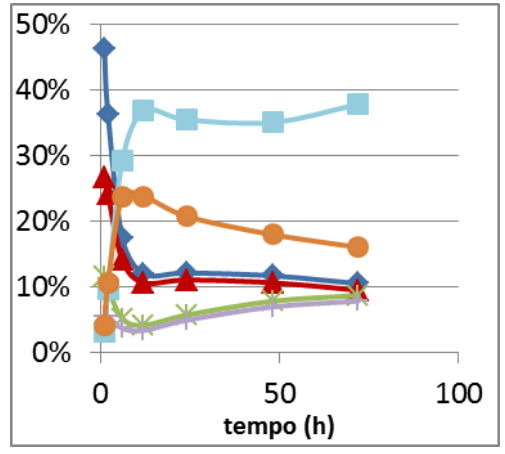

(c)

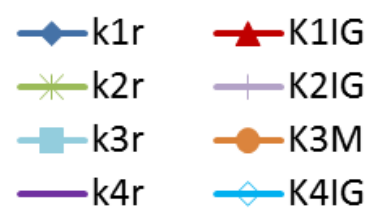

Figura 1 - Parâmetros que apresentam maior influência sobre os perfis temporais de celulose (a), glicose (b), celobiose (c), hemicelulose (d), e xilose (e).

$\mathrm{Na}$ Figura 1, a ausência de alguns parâmetros como $K_{I I G 2}, K_{I I X}, K_{2 I G 2}, K_{2 I X}, K_{3 I G}, K_{3 I X}$, $K_{4 I G 2}$ e $K_{4 I X}$, se deve ao fato de que o $S_{i}$ destes parâmetros apresenta valor ínfimo ou nulo, ou seja, no cenário experimental estudado tais parâmetros possuem baixa ou nenhuma influencia sobre a predição do modelo. Com relação aos parâmetros com maior influência no modelo $\left(k_{l r}, K_{l I G}, k_{2 r}, K_{2 I G}, k_{3 r}, K_{3 M}, k_{4 r}, K_{4 I G}\right)$, destacam-se os parâmetros $k_{2 r}$ e $K_{2 I G}$, cujos $S_{i}$ 


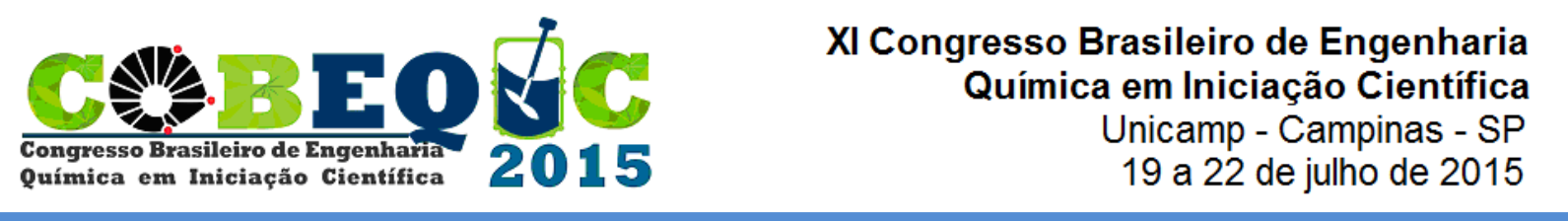

apresentam comportamento expressivo para todas as variáveis analisadas, sobretudo a celulose e a glicose. Também merecem destaques os parâmetros $k_{i r}(i=1, \ldots, 4)$ que apresentam os maiores valores de $S_{i}$, o que indica que no cenário experimental estudado este subconjunto de parâmetros são os que mais afetam as variáveis de resposta do modelo. Tais resultados demonstram a acurácia do método, visto que em um modelo cinético o conhecimento das taxas de reação é essencial para a qualidade da predição.

\section{CONCLUSÃO}

A análise de sensibilidade permitiu identificar o conjunto parâmetros que apresentam maior influência sobre a predição do modelo para o cenário experimental estudado, permitindo diferenciar quantitativamente a influência de tais parâmetros. Assim, esta ferramenta demonstra ser muito útil para os estudos de modelagem, bem como em problemas de identificabilidade de parâmetros, em que estima-se o conjunto de parâmetros selecionados quando a informação experimental disponível não é suficiente em qualidade e/ou quantidade para estimação de todos os parâmetros do modelo.

\section{AGRADECIMENTOS}

Os autores agradecem ao PEQ-COPPE/UFRJ e ao PIBIC-CNPQ, pelo auxílio financeiro.

\section{APÊNDICE}

Os elementos do modelo matemático estudado são descritos a seguir:

$\begin{array}{ccc}\text { Variável } & \text { Unidade } & \text { Descrição } \\ C & \mathrm{~g} / \mathrm{L} & \text { Concentração de celulose } \\ E_{B} & \mathrm{~g} / \mathrm{L} & \text { Concentração de enzima adsorvida no substrato } \\ E_{B C} & \mathrm{~g} / \mathrm{L} & \text { Concentração de enzima adsorvida na celulose } \\ E_{B H} & \mathrm{~g} / \mathrm{L} & \text { Concentralçao de enzima adsorvida no na hemicelulose } \\ E_{F} & \mathrm{~g} / \mathrm{L} & \text { Concentração de enzima livre } \\ E_{\max } & \mathrm{g} / \mathrm{kg} & \text { Concentração máxima de adsorção da enzima no subtrato } \\ E_{T} & \mathrm{~g} / \mathrm{L} & \text { Concentração total de enzima } \\ G & \mathrm{~g} / \mathrm{L} & \text { Concentração de glicose } \\ G 2 & \mathrm{~g} / \mathrm{L} & \text { Concentração de celobiose } \\ H & \mathrm{~g} / \mathrm{L} & \text { Concentração de hemicelulose } \\ K_{3 M} & \mathrm{~g} / \mathrm{kg} & \text { Constante da taxa de reação } \\ k_{3 r} & 1 / \mathrm{h} & \text { Constante de saturac̃ão do substrato (celobiose) } \\ K_{a d} & \mathrm{~L} / \mathrm{g} & \text { Constante de dissociação da adsorção/desorção da enzima } \\ K_{i I G} & \mathrm{~g} / \mathrm{kg} & \text { Constante de inibição para glicose }(i=1, \ldots, 4)\end{array}$




$\begin{array}{ccc}K_{i I G 2} & \mathrm{~g} / \mathrm{kg} & \text { Constante de inibição para celobiose }(i=1,2,4) \\ K_{i I X} & \mathrm{~g} / \mathrm{kg} & \text { Constante de inibição para xilose }(i=1, \ldots, 4) \\ k_{i r} & \mathrm{~kg} / \mathrm{g} / \mathrm{h} & \text { Constante da taxa de reação }(i=1,2,4) \\ L & \mathrm{~g} / \mathrm{L} & \text { Concentração de lignina } \\ r_{i} & 1 / \mathrm{g} / \mathrm{h} & \text { Taxa de reação }(i=1, \ldots, 4) \\ R_{S} & \text { adimensional } & \text { Reatividade do substrato } \\ S & \mathrm{~g} / \mathrm{L} & \text { Concentração de sólidos insolúveis } \\ X & \mathrm{~g} / \mathrm{L} & \text { Concentração de xilose } \\ A & \text { adimensional } & \text { Constante da reatividade do substrato com o grau de hidrólise }\end{array}$

\section{REFERÊNCIAS}

ANGARITA, J. D. M. Modelagem Cinética da Hidrólise Enzimática da Palha de Cana-deAçúcar Pré-tratada Hidrotermicamente. Dissertação de Mestrado, PEQ-COPPE/UFRJ, Rio de Janeiro, 2014.

BÉGUIN, P.; AUBERT, J. P. The biological degradation of cellulose. FEMS Microbiology Review, v. 13, p. 25-58, 1994.

CÂMARA, M. M. Modelagem e simulação da hidrólise de bagaço de cana pré-tratado com peróxido de hidrogênio em meio alcalino. Dissertação de Mestrado, UEM, Maringá, Paraná, 2012.

HAHN-HÄGERDAL B.; GALBE M.; GORWA-GRAUSLUND M. F.; LIDÉN G.; ZACCHI G. Bio-ethanol - the fuel of tomorrow from the residues of today. Trends in Biotechnology, v. 24, p 549-556, 2006.

HODGE D. B.; KARIM M. N.; SCHELL D. J.; MCMILLAN J. D. Model-based fed-batch for high-solids enzymatic cellulose hydrolysis. Applied Biochemistry and Biotechnology, v. 152, p 88-107, 2009.

KADAM K. L.; RYDHOLM E. C.; MCMILLAN J. D. Development and validation of a kinetic model for enzymatic saccharification of lignocellulosic biomass. Biotechnol Progress, v. 20, p. 698-705, 2004.

SALTELLI, A.; RATTO, M. Global Sensitivity Analysis. The primer. Chichester: John Wiley \& Sons, Ltd, 2008.

SOBOL, I.M. A Primer for the Monte Carlo Method. Boca Raton: CRC Press LLC, 1991.

SUN, Y.; CHENG, J. Hydrolysis of lignocellulosic materials for ethanol production: a review. Bioresource Technology, v. 83, p. 1-11, 2002. 